\title{
A simple mathematical analysis of the limitations to inferring feeding behavior of zooplankton from gut content
}

\author{
Hans G. Dam*, William T. Peterson**, Akira Okubo \\ Marine Sciences Research Center, State University of New York, Stony Brook, New York 11794-5000, USA
}

\begin{abstract}
The instantaneous amount of food in an animal's gut, or gut content $(G)$, is a function of its ingestion rate $(I)$ minus its gut evacuation rate $(E)$. We present simple models describing the short-term (minutes to hours) dynamics of $G$, incorporating $I$ and $E$ simultaneously. The models apply to situations in which animals start feeding after a period of starvation. Two possibilities are considered: (1) Both $I$ and $K$ (the coefficient of gut evacuation) remain constant during the feeding period. (2) $K$ remains constant, but $I$ decreases, after satiation, from the initial value $\left(I_{\mathrm{o}}\right)$ to an equilibrium value $\left(I_{\mathrm{e}}\right)$. Results from the models show that although gut content is usually asymptotic with time, the shapes of the curves vary considerably depending on the relationship between $I_{\mathrm{o}}$ and $K$ in the first instance, and $I_{\mathrm{o}}, I_{\mathrm{e}}$ and $K$ in the second instance. The relationship between the time of first defecation $\left(t_{1}\right)$ and the gut passage time $\left(K^{-1}\right)$ is critical in these models. Results from the models are compared to data from published experiments in which gut content of copepods was monitored at intervals of minutes to hours. We conclude that short-term variability in feeding behavior may not always be inferred solely from changes in gut content with time.
\end{abstract}

\section{INTRODUCTION}

Gut content indices have been applied extensively to infer feeding rates of aquatic herbivores (see review by Baars \& Helling 1985) and predators (references in Murtaugh 1984a). Time-dependent changes in gut content have also been used to infer variability in feeding behavior of zooplankton (e.g. Mackas \& Burns, 1986). However, the rate of change $(\mathrm{d} G / \mathrm{d} t)$ of a tracer (e.g. plant pigment, biogenic silica, ${ }^{68} \mathrm{Ge}$ ) in the gut of an animal must be considered a function of the ingestion rate $(I)$, the gut evacuation rate $(E)$ and the rate at which the tracer is absorbed or destroyed $(U)$ (e.g. Wang \& Conover 1986). This approach has been successfully applied by fisheries scientists interested in estimating daily rations of fish from gut content (Eggers 1977, Elliot \& Persson 1978, Jobling 1981).

Following the approach of Elliot \& Persson (1978), we present 2 simple models describing changes in gut

Present addresses:

- Department of Marine Sciences, University of Connecticut, Groton, Connecticut 06340, USA

- NOAA, Center for Ocean Analysis and Prediction, 2560 Garden Road, Suite 101, Monterey, California 93940, USA content with time (minutes to hours) incorporating simultaneously ingestion and gut evacuation rates. In developing these models, we have planktonic copepods in mind; thus, our discussion and examples will deal with planktonic copepods. For simplicity, we assume in this case that there is no absorption or destruction (cf. Conover et al. 1986, review in Dam \& Peterson 1988) of the tracer in the animal's gut; therefore, the term $U$ above is ignored. We explore the case of constant ingestion rate versus declining ingestion rate after satiation. Because the models apply to animals that have experienced a period of starvation, they may be used to examine the dynamics of feeding behavior not only of laboratory animals, but also of those that experience a severely patchy food environment in nature, such as herbivores that perform extensive diel vertical migration in the ocean.

\section{FORMULATION OF THE MODELS}

Our models describe the instantaneous ingestion rate, $I(t)$, in terms of the rate of change in gut content, $\mathrm{d} G / \mathrm{d} t$, and the gut evacuation rate, $E(t)$; i.e. 


$$
I(t)=\mathrm{d} G(t) / \mathrm{d} t+E(t)
$$

where we assume that

$$
E(t)= \begin{cases}0, & t<t_{1} \\ K G(t), & t \geq t_{1}\end{cases}
$$

where $t_{1}=$ time at which defecation starts; $K$ is the coefficient of gut evacuation. $G$ has units of mass of tracer ind. ${ }^{-1} ; I$ has units of mass of tracer ind. ${ }^{-1}$ time $^{-1}$ and $K$ has units of time ${ }^{-1}$. It is usually assumed that $K^{-1}$ represents the gut passage time. In poststarvation feeding experiments, defecation does not occur immediately after animals start feeding (Dagg 1983, Dam 1986); thus, we take $E(t)=0$ for $t<t_{1}$. If gut evacuation is assumed to be suitably described by exponential decay behavior (Mackas \& Bohrer 1976, Kiørboe et al. 1982, Dagg \& Wyman 1983, Kiørboe \& Tiselius 1987, Dam \& Peterson 1988, Ellis \& Small 1989), then the gut evacuation rate $(E)$ can be expressed as $E=K G(t)$ for $t \geq t_{1}$.

For $I(t)$, we consider 2 cases

Model I: $I(t)=I_{0}, \quad$ for all $t$

Model II:

$$
I(t)= \begin{cases}I_{\mathrm{o}} \quad & t<t_{1} \\ I_{\mathrm{e}}+\left(I_{o}-I_{\mathrm{e}}\right) \exp \left\{-b\left(t-t_{1}\right)\right\}, \quad t \geq t_{1}\end{cases}
$$

where $I_{\mathrm{o}}>I_{\mathrm{e}}\left(I_{\mathrm{o}}\right.$ is a constant and $I_{\mathrm{e}}$ is the equilibrium ingestion rate to which $I$ decreases after satiation) and $b$ is a positive constant. Detailed explanations of the parameters of both models are given below.

\section{Solution for $G(t)$ in Model I}

Substituting Eqs. (2) and (3) into Eq. (1) and integrating the resulting equation over time subject to the initial condition that $G(0)=0$, we obtain the following solutions

$$
G(t)= \begin{cases}I_{0} t_{1} \quad t<t_{1} \\ G_{1 \infty}+\left(G_{1}-G_{1 \infty}\right) \exp \left\{-K\left(t-t_{1}\right)\right\}, \quad t \geq t_{1}\end{cases}
$$

where $G_{1 \infty}=I_{\mathrm{o}} / K$

$$
G_{1}=I_{\mathrm{o}} t_{1}
$$

Examination of Eq. (5) shows that in the absence of defecation, changes in gut content with time are due solely to ingestion (Fig. 1). Since ingestion rate is constant, gut content increases linearly with time and the total amount of food ingested, $Q(t)$, will also increase linearly with time (Fig. 1).

After defecation has begun, the behavior of $G(t)$ depends on how gut content at the onset of defecation $\left(G_{1}\right)$ compares to the ratio of ingestion to the coefficient of gut evacuation $\left(I_{\mathrm{o}} / K=G_{1 \infty}\right)$; i.e. $G_{1 \infty}$ represents the asymptotic value of gut content and, depending upon the relative value of $G_{1}$ and $G_{1 \infty}$, the behavior of $G(t)$ after $t>t_{1}$ is different. In Fig. 1, we show 3 different cases: $G_{1}<G_{1 \infty}$ (Case 1), $G_{1}=G_{1 \infty}$ (Case 2), $G_{1}>G_{1 \infty}$ (Case 3). Examination of Eq. (7) above shows that Case 1 occurs when $t_{1}<K^{-1}$; Case 2 occurs when $t_{1}=K^{-1}$ and Case 3 occurs when $t_{1}>K^{-1}$.

\section{Solution for $G(t)$ in Model II}

In Model II we assume that once defecation starts, the ingestion rate is no longer constant, but instead decreases with time to some equilibrium rate, $I_{\mathrm{e}}$, the constant parameter $b$ represents the rate of decay of ingestion with time. The value of $I_{\mathrm{e}}$ for planktonic copepods is variable and seems to range from 0.1 to 0.5 $I_{\mathrm{o}}$ (deduced from Mullin 1963, Runge 1980, Hassett \& Landry 1988). Substituting Eqs. (2) and (4) into Eq. (1) and integrating the resulting equation, we obtain

$$
\begin{aligned}
G(\mathrm{t})= & \left\{\begin{array}{l}
I_{\mathrm{o}} t_{1} \quad t<t_{1} \\
G_{2 \infty}+\left(G_{1}-G_{2}\right) \exp \left\{-K\left(t-t_{1}\right)\right\}
\end{array}\right. \\
& +\left(G_{2}-G_{2 \infty}\right) \exp \left\{-b\left(t-t_{1}\right)\right\}, \quad t \geq t_{1}
\end{aligned}
$$

where $G_{2 \infty}=I_{\mathrm{e}} / K$

$$
G_{2}=\left(I_{\mathrm{e}} / K\right)+\left(I_{\mathrm{o}}-I_{\mathrm{e}}\right) /(K-b)
$$

Changes in gut content with time after the ingestion rate starts to decrease can be examined from Eq. (8). $G_{2 \infty}$ represents the asymptotic (equilibrium) behavior of $G(t)$ while the other 2 terms ( $K$ and $b$ exponential terms) represent the transient behavior of $G(t)$. Notice that $G_{2 \infty}<G_{1 \infty}$. By equating the time derivative of Eq.

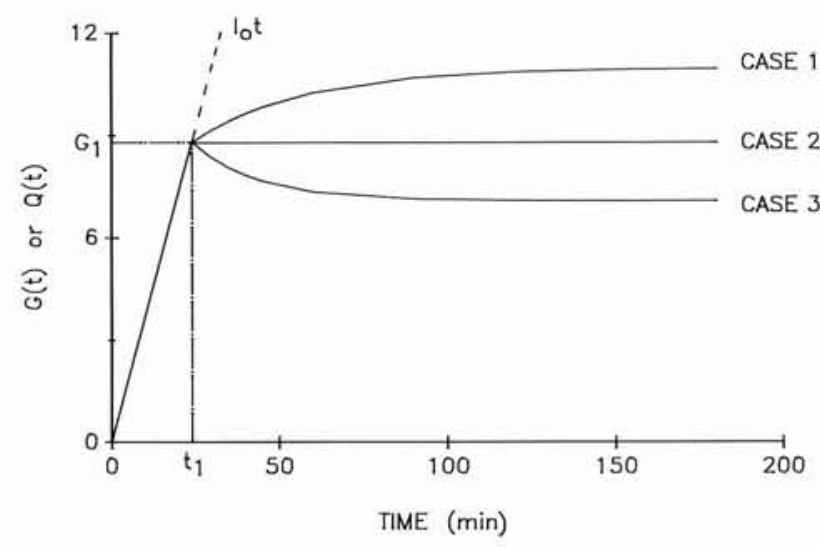

Fig. 1. Model I. Gut content, $G(t)$ (solid line), or total amount of food ingested, $Q(t)$ (solid-dashed line), vs time. Ingestion rate $\left(I_{\mathrm{o}}\right)$ and coefficient of gut evacuation $(K)$ are assumed to remain constant with time. $G(t)$ and $Q(t)$ have units of mass of tracer ind. ${ }^{-1}$ (e.g. nanogram tracer ind. ${ }^{-1}$ ). Model parameters for this example are: $I_{\mathrm{o}}=0.3666 \mathrm{ng}$ tracer ind. ${ }^{-1} \mathrm{~min}^{-1}, t_{1}=24$ $\min$; Case 1: $K=0.0374 \mathrm{~min}^{-1}\left(t_{1}<K^{-1}\right)$; Case 2: $K=0.0417$ $\min ^{-1}\left(t_{1}=K^{-1}\right)$; Case 3: $K=0.0520 \min ^{-1}\left(t_{1}>K^{-1}\right)$ 

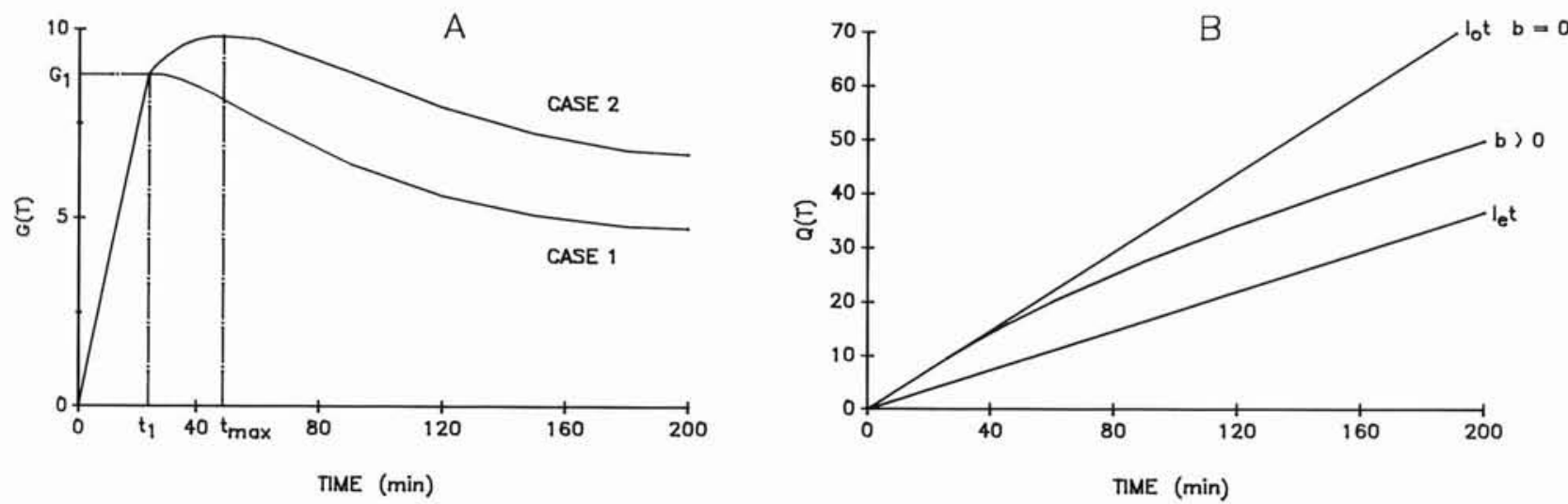

Fig. 2. Model II. (A) Gut content, $G(t)$, vs time. Ingestion rate $(I)$ is assumed to decrease with time $(t)$, with the onset of defecation, as $I=I_{\mathrm{e}}+\left(I_{\mathrm{o}}-I_{\mathrm{e}}\right) \exp \left\{-b\left(t-t_{1}\right)\right\}$, where $b$ is a constant, $I_{\mathrm{o}}$ is the ingestion rate at the onset of defecation $\left(t_{1}\right)$, and $I_{\mathrm{e}}$ is the

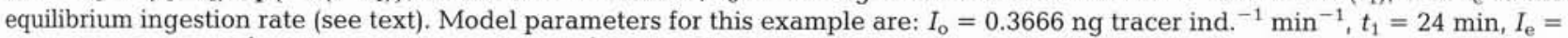
$0.5 I_{0}, b=0.020 \mathrm{~min}^{-1}$; Case 1: $K=0.04166 \mathrm{~min}^{-1}\left(G_{2}>G_{1} ; K>b ; t_{1}=K^{-1}\right)$; Case 2: $K=0.030 \min ^{-1}\left(G_{2}>G_{1} ; K>b ; t_{1}<K^{-1}\right)$. For definition of $G_{2}$ see text. (B) Total amount of food ingested, $Q(t)$, vs time. $I_{\mathrm{e}}$ as in (A). For $b>0, b=0.02$. Units of $G(t)$ and $Q(t)$ above as in Fig. 1.

(8) to 0 , we can determine when $G$ becomes maximum at $t=t_{\max }\left(t=t_{\max }>t_{1}\right)$

We thus obtain

$$
\begin{aligned}
& \exp \left\{(K-b)\left(t_{\max }-t_{1}\right)\right\} \\
& \quad=\left[K(K-b) /\left\{b\left(I_{\mathrm{o}}-I_{\mathrm{e}}\right)\right\}\right]\left(G_{2}-G_{1}\right)>1
\end{aligned}
$$

Substituting Eqs. (7) and (10) into the right-hand side of Eq. (11), we define

$$
Z=1+\left\{I_{\mathrm{o}} /\left(b\left(I_{\mathrm{o}}-I_{\mathrm{e}}\right)\right\}(K-b)\left(1-K t_{1}\right)\right.
$$

Keeping in mind that $I_{\mathrm{o}}>0, b>0$ and $I_{\mathrm{o}}>I_{\mathrm{e}}$, then if $K>b, Z>1$ only when $t_{1}<K^{-1}$, and then $t_{\max }>t_{1}$ since $\exp \left\{(K-b)\left(t_{\max }-t_{1}\right)\right\}=Z>1$. If, on the other hand, $K<b, Z<1$ only when $t_{1}<K^{-1}$, and then again $t_{\max }>t_{1}$ since $\exp \left\{(K-b)\left(t_{\max }-t_{1}\right)\right\}<1$. In summary, we can reduce the criterion of $t_{\max }>t_{1}$ to $t_{1}<K^{-1}$ regardless of the relationship between $K$ and $b$ and between $G_{1}$ and $G_{2}$.

In Fig. 2a we illustrate some of the possible behaviors of $G(t)$ for Model II. Case 1 represents the special case in which $G_{1}=G_{\max }$ gut content declines as soon as the ingestion rates starts to decrease. This case occurs when $t_{1}=K^{-1}$. Case 2 represents a case in which $G_{\max }>G_{1}$. This occurs when $t_{1}<K^{-1}$; in this particular example, $G_{2}>G_{1}$ and $K>b$.

Integrating Eq. (4) gives the total amount of food ingested as a function of time

$$
\begin{aligned}
& Q(t)=\left\{\begin{array}{l}
I_{\mathrm{o}} t_{1} \quad t<t_{1} \\
I_{\mathrm{o}} t_{1}+I_{\mathrm{e}}\left(t-t_{1}\right)
\end{array}\right. \\
& +\left\{\left(I_{\mathrm{o}}-I_{\mathrm{e}}\right) / b\right\}\left[1-\exp \left\{-b\left(t-t_{1}\right)\right\}\right], \quad t>t_{1}
\end{aligned}
$$

In the interval $0<t<t_{1}, Q(t)$ is given by $I_{\mathrm{o}} t$ since defecation is absent and the ingestion rate has not started to decrease yet. As time increases after the ingestion rate starts to decrease, $Q(t)$ tends to increase more slowly and asymptotically approaches $I_{\mathrm{e}} t$ (Fig. 2b).

\section{DISCUSSION}

The results of several published studies, in which gut content of zooplankton was monitored at short-time intervals, can be compared with the predictions of our models. For instance, the levelling off of copepod gut content with time in poststarvation feeding experiments reported by Dagg (1983), Mackas \& Burns (1986, their Fig. 4a, b), Dam (1986), and Head \& Harris (1987, their Figs. 5 \& 6 ) would fit Cases 1 and 2 of Model I and would imply that ingestion rate remained constant for the duration of the experiments. However, it would be difficult to discriminate in an experiment between Cases 1 and 2 because determining whether the curve of $G(t)$ vs time is asymptotic would require very frequent sampling. Case 3 of Model I is particularly interesting since it shows that a drop in gut content is possible even if the ingestion rate remains constant. Case 3 shows a similar behavior to the conceptual model of Mackas \& Burns (1986, their Fig. 1b), but they attribute the decline in gut content solely to a decrease of the ingestion rate.

The decline in gut content after the initial peak in poststarvation feeding experiments reported by Mackas \& Burns (1986, their Fig. 2), Head \& Harris (1987, their Figs. $7 \&$ 8) and Head (1988, her Figs. 1 \& 7) resemble the outcomes of Model II, and would suggest that the copepods reduced their ingestion rate with time. However, because we have shown that it is possible to have a significant drop of gut content with time even if the ingestion rate remains constant (our Case 3 of Model I) one must exercise caution in concluding 
that a drop in gut content always indicates a reduction of the ingestion rate (compare Fig. 1 and Fig. 2a). This is particularly important if the experiments are run for only a few hours (e.g. Dagg 1983, Dam 1986, Wang \& Conover 1986, Head \& Harris 1987) in which case a plateau in gut content after the initial drop may not be reached.

We have also shown in Model II that there exists the possibility of gut content not attaining a maximum until a time well after the ingestion rate has started to decrease; e.g. in our simulation for Case 2, maximum gut content was not achieved until almost 25 min after the ingestion rate had started to decline (Fig. 2a). Thus, under poststarvation conditions, equating the time of maximum gut content with the time at which the ingestion rate starts to decrease is not always warranted.

Whether or not ingestion rate changes with time can be tested directly by setting up experiments in which individual, or groups of, starved copepods are isolated in a series of beakers containing food solutions (labelled with an inert tracer, e.g. biogenic silica or ${ }^{68} \mathrm{Ge}$ ) and are allowed to feed for fixed periods of time. The concentration of the tracer in the guts and the fecal pellets of the copepods is then measured. This concentration represents the amount of the tracer ingested from the beginning of the experiment, $Q(t)$. A comparison of the slopes of $Q(t)$ vs time for the predefecation and postdefecation period (Fig. 2b) would allow one to detect variations in the ingestion rate. This test would assume that copepods behave somewhat synchronously while feeding, and the available data suggest that this assumption is valid for poststarvation feeding experiments (e.g. Dagg 1983, Dam 1986, Mackas \& Burns 1986).

It is important to emphasize that the above experiments must be carried out with an inert tracer. For example, it may not be possible to use plant pigments as a tracer for these experiments because of the possibility of pigment digestion/destruction either during passage through the gut or during the handling of samples (cf. recent reviews in Dam \& Peterson 1988 and Downs 1989; however, see also Pasternak \& Drits 1989 and Peterson et al. 1990).

For simplicity we have assumed in the formulation of the models that the time of first defecation, $t_{1}$, is fixed. However, since the outcomes of the model are sensitive to the value of $t_{1}$, it is important that this parameter be accurately estimated. This parameter could be estimated directly, e.g. in the type of experiment proposed above to test for difference in ingestion rates, by monitoring the time of fecal pellet appearance in the beakers containing the copepods. If copepods are feeding at food concentrations above the critical concentration (sensu Frost 1972), $t_{1}$ may be a constant. Below the critical food concentration, $t_{1}$ may vary inversely with food concentration and ingestion rate (Murtaugh $1984 a, b)$. Therefore, $t_{1}$ should be measured at different food concentrations and under different ingestion rates in experiments to test these models.

Our models predict different behaviors of $G$ vs $t$ depending on the relationship between $t_{1}$ and the gut passage time $\left(\mathrm{K}^{-1}\right)$. It would be particularly useful to examine the conditions under which $t_{1}$ would be greater than or less than the average gut passage time. For example, it is conceivable that at very low food concentrations copepods may start defecating before their guts are full (e.g. Dagg \& Walser 1986). Thus, one may observe the overshoot in gut content (Case 1 of Model I and Case 2 of Model II). If copepods were to gorge themselves, perhaps while feeding at high food concentrations after a very long period of starvation, then it would be possible that $t_{1}$ would exceed the average gut passage time. One would then observe a decline in gut content with time as in Case 3 of Model I and Case 1 of Model 2.

Another simplifying assumption made in Model II is that the ingestion rate is reduced immediately upon the time of first defecation. There is evidence that a decrease in ingestion rate is coupled to a saturation of gut processes (Nott et al. 1985, Hassett \& Landry 1988); thus, presumably the decrease in ingestion rate is related to satiation. However, whether the time of first defecation represents the onset of satiation remains an open question.

In summary, we have presented 2 models describing the time-dependent changes of gut content in zooplankton given different sets of ingestion and egestion rates. Specifically, we have shown that the decline in gut content frequently observed in short-term experiments does not necessarily reflect a decline in ingestion rate; furthermore, we have shown that interpretation of changes in gut content vs time must take into account the relationships between $I_{\mathrm{o}}, I_{\mathrm{e}}$ and $K$, and between $t_{1}$ and $K$. We conclude that inferences on changes in the feeding behavior of zooplankton cannot always be made from changes in gut content alone.

Acknowledgements. We thank Drs D. Mackas, P. Jumars, M. Landry, T. Kiørboe, and an anonymous referee for constructive criticism and suggestions, although the first 2 may not agree with all of our ideas. We are especially grateful to Dr J. E. Beyer whose comments and suggestions as a referee greatly improved the final presentation of the model. This research was supported by Grants-in-Aid from the LernerGray Fund for Marine Research of the American Museum of Natural History and Sigma Xi Foundation awarded to H. G. Dam and Grant \# NA86AADSG045 from New York Sea Grant Institute awarded to W. T. Peterson. This paper was written while H. Dam held a postdoctoral appointment at HPL. This is contribution 762 of MSRC, SUNY at Stony Brook and 2150 of CEES, University of Maryland. 


\section{LITERATURE CITED}

Baars, M. A., Helling, G. R. (1985). Methodical problems in the measurement of phytoplankton ingestion rate by gut fluorescence. Hydrobiol. Bull. 19: 81-88

Conover, R. J., Roy, S. J., Wang, R. (1986). Probable loss of chlorophyll-derived pigments during passage through the gut of zooplankton, and some of the consequences. Limnol. Oceanogr. 31: 878-887

Dagg, M. J. (1983). A method for the determination of copepod feeding rate during short time intervals. Mar. Biol. 75: 63-67

Dagg, M. J., Wyman, K. D. (1983). Natural ingestion rates of the copepods Neocalanus plumchrus and $N$. christatus calculated from gut contents. Mar. Ecol. Prog. Ser. 13: 37-46

Dagg, M. J., Walser, W. E., Jr. (1986). The effect of food concentration in fecal pellet size in marine copepods. Limnol. Oceanogr, 31: 1066-1071

Dam, H. G. (1986). Short-term feeding of Temora Iongicornis Müller in the laboratory and the field. J. exp. mar. Biol. Ecol. 99: 149-161

Dam, H. G., Peterson, W. T. (1988). The effect of temperature on the gut clearance rate constant of planktonic copepods. J. exp. mar. Biol. Ecol. 123: 1-14

Downs, J. N. (1989). Export and production in oceanic systems: information from pheopigment, carbon and nitrogen analysis. Ph.D. thesis, University of Washington.

Eggers, D. M. (1977). Factors in interpreting data obtained by diel sampling of fish stomachs. J. Fish. Res. Bd Can. 34: 290-294

Elliot, J. M., Persson, A. L. (1978). The estimation of daily rates of food consumption for fish. J. Anim. Ecol. 47: 977-991

Ellis, S. G., Small, L. F. (1989). Comparison of gut evacuation rate of feeding and non-feeding Calanus marshallae. Mar. Biol. 103: 175-181

Frost, B. W. (1972). Effect of size and concentration of food particles on the feeding behavior of the marine planktonic copepod Calanus pacificus. Limnol. Oceanogr. 8: 805-815

Hassett, P. R., Landry, M. R. (1988). Short-term changes in feeding and digestion in Calanus pacificus at high food concentrations. Mar. Biol. 99: 63-74

Head, E. J. H. (1988). Copepod feeding behavior and the measurement of grazing rates in vivo and in vitro. Hydrobiol. 167/168: 31-41

Head, E. J. H., Harris, L. R. (1987). Copepod feeding patterns

This article was presented by Dr T. Kiørboe, Charlottenlund, Denmark before and during a spring bloom in Bedford Basin, Nova Scotia. Mar. Ecol. Prog. Ser. 40: 221-230

Jobling, M. (1981). Mathematical models of gastric emptying and the estimation of daily rates of food consumption for fish. J. Fish. Biol. 19: 245-257

Kiørboe, T., Møhlenberg, F., Nicolajsen, H. (1982). Ingestion rate and gut clearance in the planktonic copepod Centropages hamatus (Lilljeborg) in relation to food concentration and temperature. Ophelia 21: 181-194

Kiørboe, T., Tiselius, P. (1987). Gut clearance and pigment destruction in a herbivorous copepod, Acartia tonsa, and the determination of in situ grazing rates. J. Plankton Res. 9: 525-534

Mackas, D., Bohrer, R. (1976). Fluorescence analysis of zooplankton gut contents and an investigation of diel feeding patterns. J. exp. mar. Biol. Ecol. 25: 77-85

Mackas, D. L., Burns, K. E. (1986). Poststarvation feeding and swimming activity in Calanus pacificus and Metridia pacifica. Limnol. Oceanogr. 31: 383-392

Mullin, M. M. (1963). Some factors affecting the feeding of marine copepods of the genus Calanus. Limnol. Oceanogr. 8: $239-250$

Murtaugh, P. A. (1984a). Variable gut residence time: problems in inferring feeding rate from stomach fullness of a mysid crustacean. J. Plankton Res. 7: 415-420

Murtaugh, P. A. (1984b). The influence of food concentration and feeding rate on the gut residence time of Daphnia. J. Plankton Res. 7: 415-420

Nott, J. A., Corner, E. D. S., Marvin, L. J., O'Hara, S. C. M. (1985). Cyclical contributions of digestive epithelium to faecal pellet formation by the copepod Calanus helgolandicus. Mar. Biol. 89: 271-279

Pasternak, A. F., Drits, A. V. (1988). Possible degradation of chlorophyll-derived pigment during gut passage of herbivorous copepods. Mar. Ecol. Prog. Ser. 49: 187-190

Peterson, W., Painting, S., Barlow, R. (1990). Feeding rates of Calanoides carinatus: a comparison of five methods including evaluation of the gut fluorescence method. Mar. Ecol. Prog. Ser. 63: 85-92

Runge, J. A. (1980). Effects of hunger and season on the feeding behavior of Calanus pacificus, Limnol. Oceanogr. 25: $134-145$

Wang, R., Conover, R. J. (1986). Dynamics of gut pigment in the copepod Temora longicornis and the determination of in situ grazing rates. Limnol. Oceanogr. 31: 867-877.

Manuscript first received: September 6, 1989

Revised version accepted: October 19, 1990 